\title{
ON THE CONVERGENCE OF THE BHATTACHARYYA BOUNDS IN THE MULTIPARAMETRIC CASE
}

Abstract. Shanbhag $(1972,1979)$ showed that the diagonality of the Bhattacharyya matrix characterizes the set of normal, Poisson, binomial, negative binomial, gamma or Meixner hypergeometric distributions. In this note, using Shanbhag's techniques, we show that if a certain generalized version of the Bhattacharyya matrix is diagonal, then the bivariate distribution is either normal, Poisson, binomial, negative binomial, gamma or Meixner hypergeometric. Bartoszewicz (1980) extended the result of Blight and Rao (1974) to the multiparameter case. He gave an application of this result when independent samples come from the exponential distribution, and also evaluated the generalized Bhattacharyya bounds for the best unbiased estimator of $P(Y<X)$. We show that there are misprints in these results, give corrections and obtain the generalized Bhattacharyya bounds for the variance of the minimum variance unbiased estimator of $P(Y<X)$ when independent observations are taken from a normal or geometric distribution.

1. Introduction. Seth (1949) proved that the Bhattacharyya matrices for certain exponential families of distributions are diagonal. Shanbhag $(1972,1979)$ showed that if the $3 \times 3$ Bhattacharyya matrix is diagonal, then the family is either normal, Poisson, binomial, negative binomial, gamma or Meixner hypergeometric. Bartoszewicz (1980) proved that under some assumptions the generalized Bhattacharyya matrix is diagonal. (For the definition of the generalized Bhattacharyya matrix, see $\S 2$.) We show that if the generalized Bhattacharyya matrix is diagonal, then the bivariate distribution family is either normal, Poisson, binomial, negative binomial, gamma or Meixner hypergeometric. Blight and Rao (1974) considered the Bhat-

1991 Mathematics Subject Classification: Primary 62E10, 62H10; Secondary 60E05.

Key words and phrases: characterizations, Bhattacharyya bounds, diagonal of covariance matrix, Seth-Shanbhag results, exponential family, bivariate distributions, MVUE. 
tacharyya bounds corresponding to the variance of the minimum variance unbiased estimator (MVUE) of a function $\tau(\theta)$ of the parameter $\theta$ when the sampling distribution is a member of an exponential family with density $f(t ; \theta)$, which has the property

$$
\frac{\partial}{\partial \theta} \log f(t ; \theta)=V^{-1}(\theta)(t-\theta),
$$

where $V(\theta)=c_{0}+c_{1} \theta+c_{2} \theta^{2}$, for some constants $c_{0}, c_{1}$ and $c_{2}$. Using certain results of Seth (1949) and Shanbhag (1972), they showed that, under some regularity conditions, the Bhattacharyya bounds converge to the variance itself. They also provided a table computing the Bhattacharyya function (the $(i, i)$ th element of the Bhattacharyya matrix) explicitly for all exponential family distributions except the Meixner hypergeometric distribution. Alzaid (1987) more recently showed that the Bhattacharyya function for this distribution equals $\left[\left\{\varrho^{2}\left(1+\theta^{2}\right)\right\}^{-r} \Gamma(\varrho+r) r ! / \Gamma(\varrho)\right]$, where $\varrho$ is the parameter that appears in the expression for the density of the Meixner hypergeometric distribution which is as follows:

$$
f(x ; \theta)=(\cos \alpha)^{\varrho} \frac{2^{\varrho-2}}{\pi \Gamma(\varrho)} e^{\alpha x} \Gamma\left(\frac{\varrho}{2}+\frac{i x}{2}\right) \Gamma\left(\frac{\varrho}{2}-\frac{i x}{2}\right), \quad x \in \mathbb{R}
$$

(cf. Shanbhag (1979)).

Using their result, Blight and Rao also gave the Bhattacharyya bounds for the variance of the MVUE with examples from negative binomial and exponential distributions. Apparently, the same result was rediscovered by Khan (1984). Bartoszewicz (1980) extended the result of Blight and Rao to the multiparameter case. He also gave an application of this result when independent samples are taken from the exponential distribution, and evaluated numerically the values of the first four generalized Bhattacharyya bounds for the best unbiased estimator of $P(Y<X)$. In a practical situation, one views $X$ as the strength of a component, subjected to a stress $Y$. This model was first considered by Birnbaum (1956). Unfortunately, some of Bartoszewicz's (1980) results in this direction happen to have misprints. Here we give corrections to these results and obtain the generalized Bhattacharyya bounds for the variance of the MVUE of $P(Y<X)$, when independent samples are taken from a normal or geometric distribution. Ghosh and Sathe (1987) proved that for all estimable $\tau$ and all multiparameter exponential families, the Bhattacharyya bounds converge to the variance of the MVUE and an example is worked out where $\tau$ is a function of interest in reliability theory. This result is a particular case of Bartoszewicz's results and the example had already appeared in Bartoszewicz (1980). Here we consider the case of the family on $\mathbb{R}^{2}$ with two parameters. Extension to the general case is then easily seen. 
2. Diagonality of the generalized Bhattacharyya matrix. Suppose the random vector $\mathbf{X}\left(\equiv X^{(\theta)}\right)=\left(X_{1}, \ldots, X_{n}\right)$ has a joint probability density function

$$
f(\mathbf{x} ; \theta)=f\left(x_{1}, \ldots, x_{n} ; \theta_{1}, \ldots, \theta_{r}\right),
$$

with respect to a $\sigma$-finite measure $\mu$. Let $k>1$ be an integer, and $\mathbf{i}=$ $\left(i_{1}, \ldots, i_{r}\right), 0 \leq i_{j}, 0<i_{1}+\ldots+i_{r} \leq k$, and $\mathbf{i}^{*}=\left(i_{1}^{*}, \ldots, i_{r}^{*}\right)$ with similar properties.

We make the following assumptions:

(i) The function $f(\mathbf{x} ; \theta)$ and an estimable function $\tau(\theta)$ have all partial derivatives with respect to $\theta_{1}, \ldots, \theta_{r}$ of order up to $k$,

$$
f^{(\mathbf{i})}=\frac{\partial^{i_{1}+\ldots+i_{r}} f(\mathbf{x} ; \theta)}{\partial \theta_{1}^{i_{1}} \ldots \partial \theta_{r}^{i_{r}}}, \quad \tau^{(\mathbf{i})}=\frac{\partial^{i_{1}+\ldots+i_{r}} \tau(\theta)}{\partial \theta_{1}^{i_{1}} \ldots \partial \theta_{r}^{i_{r}}} .
$$

(ii) The expectations

$$
J\left(\mathbf{i}, \mathbf{i}^{*} ; \theta\right)=E_{\theta}\left\{\frac{f^{(\mathbf{i})}}{f(\mathbf{x} ; \theta)} \cdot \frac{f^{\left(\mathbf{i}^{*}\right)}}{f(\mathbf{x} ; \theta)}\right\}
$$

exist and are finite. The matrix with elements $J\left(\mathbf{i}, \mathbf{i}^{*} ; \theta\right)$ is called the generalized Bhattacharyya matrix. The inverse matrix $\left\|J^{*}\left(\mathbf{i}, \mathbf{i}^{*} ; \theta\right)\right\|=\left\|J\left(\mathbf{i}, \mathbf{i}^{*} ; \theta\right)\right\|^{-1}$ exists.

(iii) The function $\widehat{\tau} f(\mathbf{x} ; \theta)$ is differentiable in $\theta_{1}, \ldots, \theta_{r}$ under the integral with respect to $\mathbf{x}$ at least $k$ times, where $\widehat{\tau}$ is an unbiased estimator of $\tau(\theta)$.

Bhattacharyya (1947) proved that

$$
\operatorname{Var}(\widehat{\tau}) \geq \sum \tau^{(\mathbf{i})} \tau^{\left(\mathbf{i}^{*}\right)} J^{*}\left(\mathbf{i}, \mathbf{i}^{*} ; \theta\right),
$$

where the summation is running over all $\mathbf{i}, \mathbf{i}^{*}$. The right hand side is called the $k$ th generalized Bhattacharyya bound.

Assume the following regularity conditions:

(I) $\theta=\left(\theta_{1}, \theta_{2}\right) \in \Omega=\Omega_{1} \times \Omega_{2}$, where $\Omega_{i}(i=1,2)$ are open intervals on the real line.

(II) $\mathbf{T}=\left(T_{1}, T_{2}\right)$ is a random vector, where $T_{i}$ are independent random variables belonging to an exponential family with the property

$$
\frac{\partial}{\partial \theta_{i}} \log f_{i}\left(t_{i} ; \theta_{i}\right)=V_{i}^{-1}\left(\theta_{i}\right)\left(t_{i}-\theta_{i}\right)
$$

where $V_{i}\left(\theta_{i}\right)=c_{0}^{(i)}+c_{1}^{(i)} \theta_{i}+c_{2}^{(i)} \theta_{i}^{2}$, for some constants $c_{0}^{(i)}, c_{1}^{(i)}, c_{2}^{(i)}(i=1,2)$.

(III) The density $f(\mathbf{t} ; \theta)$ can be differentiated with respect to $\theta_{1}, \theta_{2}$ under the integral with respect to $\mathbf{t}$ any number of times. 
Under the above conditions, Bartoszewicz (1980) proved that, if $f(\mathbf{t} ; \theta)$ is of the form $f(\mathbf{t} ; \theta)=f_{1}\left(t_{1} ; \theta_{1}\right) f_{2}\left(t_{2} ; \theta_{2}\right)$, then the generalized Bhattacharyya matrix is diagonal. Shanbhag $(1972,1979)$ showed this family to be equivalent within a linear transformation to the family composed of the normal, Poisson, binomial, negative binomial, gamma and Meixner hypergeometric distributions.

TheOREM 1. Let $\mathbf{X}=\left(X_{1}, X_{2}\right)$ be a random vector, where $X_{i}$ are independent r.v.'s having probability density function of the form

$$
f\left(x_{i} ; \theta_{i}\right)=\exp \left\{x_{i} g\left(\theta_{i}\right)\right\} \psi_{i}\left(x_{i}\right) / \beta_{i}\left(\theta_{i}\right) .
$$

If the above conditions are satisfied and the generalized Bhattacharyya matrix is diagonal, then the bivariate distribution $\mathbf{X}^{*}=\left(X_{1}^{*}, X_{2}^{*}\right)$ is either normal, or Poisson, or binomial, or negative binomial, or gamma, or Meixner hypergeometric, for some linear transforms $X_{1}^{*}$ of $X_{1}$ and $X_{2}^{*}$ of $X_{2}$.

Proof. Since the generalized Bhattacharyya matrix is diagonal, its $\left(\mathbf{i}, \mathbf{i}^{*}\right)$ th element is

$$
J\left(\mathbf{i}, \mathbf{i}^{*}\right)= \begin{cases}\left\{J_{i_{1}}^{(1)}\right\}^{2}\left\{J_{i_{2}}^{(2)}\right\}^{2} & \text { if } i_{1}=i_{1}^{*} \text { and } i_{2}=i_{2}^{*}, \\ 0 & \text { otherwise }\end{cases}
$$

where

$$
\left\{J_{i_{j}}^{(j)}\right\}^{2}=E\left\{\frac{1}{f\left(X_{j} ; \theta\right)} \frac{\partial^{i_{j}} f\left(X_{j} ; \theta\right)}{\partial \theta_{j}^{i_{j}}}\right\}^{2}, \quad j=1,2,
$$

are the Bhattacharyya functions (see Bhattacharyya (1947)).

From Shanbhag (1972), it easily follows that

$$
\begin{aligned}
E\left[X_{i}\right] & =c_{0}^{(i)}+c_{1}^{(i)} \theta_{i}, \\
E\left[X_{i}^{2}\right] & =c_{11}^{(i)}+c_{12}^{(i)} \theta_{i}+c_{22}^{(i)} \theta_{i}^{2}, \\
\operatorname{Var}\left[X_{i}^{2}\right] & =c_{13}^{(i)}+c_{23}^{(i)} \theta_{i}+c_{33}^{(i)} \theta_{i}^{2}, \quad i=1,2,
\end{aligned}
$$

for some constants $c_{0}^{(i)}, c_{1}^{(i)}, c_{r s}^{(i)}(r, s=1,2,3)$ not depending on $\theta_{i}$. Next we identify the different cases which lead us to results of the type of Shanbhag (1972, 1979), but in the bivariate case.

Remark. From Shanbhag (1972, 1979), we may note that the Bhattacharyya functions (i.e., the diagonal elements of the Bhattacharyya matrices) are given by

$$
\left\{J_{i}(\theta)\right\}^{2}=\left[g^{\prime}(\theta)\right]^{i} \frac{d^{i}}{d \theta^{i}} E\left[(\phi(\mathbf{X}))^{i}\right]
$$

where the joint distribution of $\left(X_{1}, \ldots, X_{n}\right)$ is absolutely continuous (with respect to a measure $\mu$ ) with density 


$$
f(\mathbf{x} ; \theta)=\exp \{\phi(\mathbf{x}) g(\theta)\} \psi(\mathbf{x}) / \beta[g(\theta)],
$$

and the distribution is any of the distributions characterized by Shanbhag (1979). This result yields in particular the expressions for the diagonal elements of the Bhattacharyya matrices relative to the distributions in question given by Blight and Rao (1974) and Alzaid (1983).

3. Applications of Bhattacharyya bounds for the variance of the MVUE of $P(Y<X)$. The problem of estimating $P(Y<X)$ when $X$ and $Y$ are independent r.v.'s has been considered by several authors. The variance of the MVUE of $P(Y<X)$ in the case when samples are taken independently from exponential distributions was derived by Bartoszewicz (1980), among others. Corrections to these results together with further applications on the Bhattacharyya bounds for the variance of the MVUE of $P(Y<X)$ are given here.

3.1. Comments on Bartoszewicz's results with corrections. Tong (1974, 1975) and Johnson (1975) obtained the UMVUE of the probability when $X$ and $Y$ are independent one-parameter exponential r.v.'s. Kelley et al. (1976) derived the variance of UMVUE of the estimator (corresponding to the Bhattacharyya bounds), considering the case when one of the parameters is known. As mentioned earlier, Bartoszewicz (1980) found the variance of the MVUE of $P(Y<X)$ in the case when samples are taken independently from exponential distributions and also evaluated the generalized Bhattacharyya bounds for the best unbiased estimator of $P(Y<X)$. His formula, for the variance of the MVUE $(\operatorname{Var}(\widehat{P}))$, happens to be incorrect; this could be due to printing errors. The following is a corrected version of the formula in question:

$$
\begin{aligned}
& \operatorname{Var}(\widehat{P})=\sum_{k=1}^{\infty} \sum_{j=0}^{k} \frac{\left(\begin{array}{c}
k \\
j
\end{array}\right)^{2}}{\left(\begin{array}{c}
n+j-1 \\
j
\end{array}\right)\left(\begin{array}{c}
m+k-j-1 \\
k-j
\end{array}\right)} \\
& \quad \times\left(\frac{j(1+\varrho)-k \varrho}{k}\right)^{2} \frac{\varrho^{2 j}}{(1+\varrho)^{2(k+1)}}
\end{aligned}
$$

where $\varrho=\theta_{1} / \theta_{2}$ and $\theta_{1}, \theta_{2}$ are the expected values of $X$ and $Y$ respectively.

Also the correct table for the values of the first four generalized Bhattacharyya bounds for the best unbiased estimator of $P(Y<X)$ is as follows. It can be seen from the table that the convergence is fairly fast in all the cases. 
TABLE 1

Generalized Bhattacharyya bounds for the best unbiased estimator of $P(Y<X)$ in the exponential case

\begin{tabular}{rcccc|rrrrr}
\hline & \multicolumn{5}{c|}{$n=m=5$} & \multicolumn{5}{c}{$n=m=10$} \\
\cline { 2 - 9 }$\varrho$ & $B_{1}$ & $B_{2}$ & $B_{3}$ & $B_{4}$ & \multicolumn{1}{c}{$B_{1}$} & $B_{2}$ & $B_{3}$ & \multicolumn{1}{c}{$B_{4}$} \\
\hline .25 & 10.24 & 11.77 & 12.13 & 12.24 & 5.12 & 5.53 & 5.58 & 5.60 \\
.50 & 19.75 & 21.80 & 22.17 & 22.26 & 9.88 & 10.43 & 10.49 & 10.50 \\
.75 & 23.99 & 26.08 & 26.42 & 26.49 & 12.00 & 12.56 & 12.61 & 12.62 \\
1.00 & 25.00 & 27.08 & 27.41 & 27.48 & 12.50 & 13.07 & 13.12 & 13.12 \\
\cline { 2 - 9 } & \multicolumn{4}{c}{$n=5, m=10$} & \multicolumn{4}{c}{$n=20, m=10$} \\
& $B_{1}$ & $B_{2}$ & $B_{3}$ & $B_{4}$ & $B_{1}$ & $B_{2}$ & $B_{3}$ & $B_{4}$ \\
\hline .25 & 7.68 & 8.23 & 8.30 & 8.32 & 3.84 & 4.19 & 4.24 & 4.25 \\
.50 & 14.81 & 15.69 & 15.79 & 15.80 & 7.41 & 7.86 & 7.91 & 7.91 \\
.75 & 17.99 & 19.11 & 19.24 & 19.26 & 9.00 & 9.41 & 9.45 & 9.45 \\
1.00 & 18.75 & 20.08 & 20.25 & 20.28 & 9.37 & 9.73 & 9.76 & 9.76 \\
\hline
\end{tabular}

$B_{i}, i=1,2,3,4$, multiplied by $10^{3}$.

R e mark. One can see that the values in Bartoszewicz's Table 1 are the same or almost the same in the great majority of cases.

3.2. Further applications. Here we derive the variance of the MVUE of $P(Y<X)$ when independent samples are taken from a normal or geometric distribution and also evaluate the generalized Bhattacharyya bounds for the best unbiased estimator of $P(Y<X)$.

1. Normal case. Let $X$ and $Y$ be independent normal variables and assume that independent samples $\left(X_{1}, \ldots, X_{n}\right)$ and $\left(Y_{1}, \ldots, Y_{m}\right)$ are at hand. Then the probability that $Y$ is less than $X$ is given by

$$
R=P(Y<X)=\Phi\left(\frac{\mu_{1}-\mu_{2}}{\sqrt{\sigma_{x}^{2}+\sigma_{y}^{2}}}\right),
$$

where $\Phi()$ is the standard normal d.f. The problem of estimating $R$ has been considered by Church and Harris (1970), Downton (1973), Owen et al. (1964), Govindarajulu (1968) and more recently, Reiser and Guttman $(1986,1987)$. We will consider the case where $\sigma_{x}^{2}, \sigma_{y}^{2}$ are known and will, without loss of generality, take $\sigma_{x}^{2}=\sigma_{y}^{2}=1$. Thus

$$
\tau(\mu)=P(Y<X)=\Phi\left(\frac{\mu_{1}-\mu_{2}}{\sqrt{2}}\right) .
$$

Bartoszewicz (1980) showed that, under certain regularity conditions, the 
variance of $\widehat{R}$, the MVUE of the function $\tau(\mu)$, is given by

$$
\operatorname{Var}(\widehat{R})=\sum_{k=1}^{\infty} \sum_{j=0}^{k}\left\{\frac{\partial^{k} \tau(\mu)}{\partial \mu_{1}^{j} \partial \mu_{2}^{k-j}} \cdot \frac{1}{J_{j}^{(1)}\left(\mu_{1}\right) J_{k-j}^{(2)}\left(\mu_{2}\right)}\right\}^{2} .
$$

Blight and Rao (1974) derived the Bhattacharyya functions for the normal distribution

$$
\left\{J_{i}^{(1)}\left(\mu_{1}\right)\right\}^{2}=n^{i} i \text { ! }
$$

and similarly

$$
\left\{J_{i}^{(2)}\left(\mu_{2}\right)\right\}^{2}=m^{i} i !
$$

It is easy to verify that

$$
\begin{aligned}
\frac{\partial^{i_{1}+i_{2}}}{\partial \mu_{1}^{i_{1}} \partial \mu_{2}^{i_{2}}} \tau(\mu)= & \frac{(-1)^{i_{1}+1}}{2^{i_{1}+i_{2}} \sqrt{\pi}} \exp \left\{-\frac{1}{4}\left(\mu_{1}-\mu_{2}\right)^{2}\right\} \\
& \times \sum_{r=0}^{\infty}(-1)^{r} \frac{\left(i_{1}+i_{2}-1\right)^{(2 r)}}{r !}\left(\mu_{1}-\mu_{2}\right)^{i_{1}+i_{2}-1-2 r},
\end{aligned}
$$

where $x^{(2 r)}=x(x-1) \ldots(x-2 r+1)$ and $x^{(0)}=1$.

Hence

$$
\begin{aligned}
\operatorname{Var}(\widehat{R})= & \sum_{k=1}^{\infty} \sum_{j=0}^{k}\left\{\frac{(-1)^{j_{1}+1}}{2^{k} \sqrt{\pi}} \exp \left\{-\frac{1}{4}\left(\mu_{1}-\mu_{2}\right)^{2}\right\}\right\}^{2} \\
& \times\left\{\sum_{r=0}^{\infty}(-1)^{r} \frac{(k-1)^{(2 r)}}{r !}\left(\mu_{1}-\mu_{2}\right)^{k-1-2 r}\right\}^{2} \frac{1}{n^{j} m^{k-j} j !(k-j) !} .
\end{aligned}
$$

Putting $\delta=\mu_{1}-\mu_{2}$, we get

$$
\begin{aligned}
\operatorname{Var}(\widehat{R})= & \frac{1}{\pi} \exp \left\{-\frac{1}{2} \delta^{2}\right\} \sum_{k=1}^{\infty} \sum_{j=0}^{k} \frac{1}{n^{j} m^{k-j} j !(k-j) !} \\
& \times\left\{\frac{(-1)^{j_{1}+1}}{2^{k}} \sum_{r=0}^{\infty}(-1)^{r} \frac{(k-1)^{(2 r)}}{r !} \delta^{k-1-2 r}\right\}^{2} .
\end{aligned}
$$

The table below gives the values of the first four generalized Bhattacharyya bounds for $\delta=0.5,1.0,1.5,2.0$ and $n=m=5 ; n=m=10 ; n=5$, $m=10 ; n=20, m=10$. Again, it can be seen from the table that the series converges very quickly. 
TABLE 2

Generalized Bhattacharyya bounds for the best unbiased estimator of $P(Y<X)$ in the normal case

\begin{tabular}{ccccc|cccc}
\hline & \multicolumn{4}{c|}{$n=m=5$} & \multicolumn{4}{c}{$n=m=10$} \\
\cline { 2 - 9 }$\delta$ & $B_{1}$ & $B_{2}$ & $B_{3}$ & $B_{4}$ & $B_{1}$ & $B_{2}$ & $B_{3}$ & $B_{4}$ \\
\hline 0.5 & 10.24 & 31.23 & 31.38 & 31.39 & 14.03 & 14.82 & 14.84 & 14.84 \\
1.0 & 19.20 & 19.29 & 19.33 & 19.33 & 9.649 & 9.649 & 9.653 & 9.653 \\
1.5 & 10.32 & 10.68 & 10.68 & 10.69 & 5.164 & 5.254 & 5.254 & 5.255 \\
2.0 & 4.306 & 4.790 & 4.819 & 4.820 & 2.153 & 2.274 & 2.277 & 2.277 \\
\cline { 2 - 9 } & \multicolumn{4}{c}{$n=5, m=10$} & \multicolumn{4}{c}{$n=20, m=10$} \\
& $B_{1}$ & $B_{2}$ & $B_{3}$ & $B_{4}$ & $B_{1}$ & $B_{2}$ & $B_{3}$ & $B_{4}$ \\
\hline 0.5 & 21.05 & 22.83 & 22.89 & 22.90 & 10.52 & 10.97 & 10.98 & 10.98 \\
1.0 & 14.47 & 14.47 & 14.48 & 14.48 & 7.237 & 7.237 & 7.238 & 7.238 \\
1.5 & 7.747 & 7.949 & 7.949 & 7.951 & 3.924 & 3.924 & 3.924 & 3.924 \\
2.0 & 3.229 & 3.502 & 3.514 & 3.514 & 1.614 & 1.682 & 1.684 & 1.684 \\
\hline
\end{tabular}

$B_{i}, i=1,2,3,4$, multiplied by $10^{3}$.

2. Geometric case. Let $X_{1}, \ldots, X_{n}$ be independent identically geometric r.v.'s with probability function

$$
P(X=x)=p_{1} q_{1}^{x-1}, \quad x=1,2, \ldots,
$$

and $Y_{1}, \ldots, Y_{m}$ be independent identically geometric r.v.'s with probability function

$$
P(Y=y)=p_{2} q_{2}^{y-1}, \quad y=1,2, \ldots
$$

Also let $\left(X_{1}, \ldots, X_{n}\right)$ and $\left(Y_{1}, \ldots, Y_{m}\right)$ be independent random samples. Considering $\theta_{i}=p_{i}^{-1}, i=1,2$, we get

$$
\tau(\theta)=P(Y<X)=\frac{\theta_{1}-1}{\theta_{1}+\theta_{2}-1} .
$$

We specialize now to the case of $n=m=1$. Since $(X, Y)$ is a complete sufficient statistic for $\left(\theta_{1}, \theta_{2}\right)$, the Lehmann-Scheffe theorem assures that $I_{\{Y<X\}}$ (the indicator function of the set $\left.\{Y<X\}\right)$ is the MVUE of $P(Y<$ $X)=p_{2} q_{1} /\left(p_{1}+p_{2}-p_{1} p_{2}\right)$. We have $\sigma^{2}(\widehat{\tau})=P(Y<X)-(P(Y<X))^{2}=$ $P(Y<X)(1-P(Y<X))$, where $\widehat{\tau}$ is the indicator function of $\{Y<X\}$; this is the exact expression for the variance of the MVUE. Now using the Bartoszewicz result, we can obtain the variance of $\widehat{\tau}$ and see whether the sequence of the generalized Bhattacharyya bounds converges to the variance of the MVUE. It is easily verified that

$$
\frac{\partial^{i_{1}+i_{2}}}{\partial \theta_{1}^{i_{1}} \partial \theta_{2}^{i_{2}}} \tau(\theta)=\frac{(-1)^{i_{1}+i_{2}}\left(i_{1}+i_{2}-1\right) !\left(i_{2} \theta_{1}-i_{1} \theta_{2}-i_{2}\right)}{\left(\theta_{1}+\theta_{2}-1\right)^{\left(i_{1}+i_{2}+1\right)}} .
$$


Blight and Rao (1974) derived the Bhattacharyya functions for the negative binomial distribution to be

$$
\left\{J_{i}^{(1)}(\theta)\right\}^{2}=\frac{(r+i-1) ! i !}{(r-1) !\{\theta(\theta-1)\}^{i}},
$$

so the Bhattacharyya functions for the geometric distribution are

$$
\left\{J_{i}^{(1)}\left(\theta_{1}\right)\right\}^{2}=\frac{(i !)^{2}}{\left\{\theta_{1}\left(\theta_{1}-1\right)\right\}^{i}},
$$

and similarly

$$
\left\{J_{i}^{(2)}\left(\theta_{2}\right)\right\}^{2}=\frac{(i !)^{2}}{\left\{\theta_{2}\left(\theta_{2}-1\right)\right\}^{i}} .
$$

Hence

$$
\begin{aligned}
\operatorname{Var}(\widehat{\tau})= & \sum_{k=1}^{\infty} \sum_{j=0}^{k}\left\{\frac{\partial^{k} \tau(\theta)}{\partial \theta_{1}^{j} \partial \theta_{2}^{k-j}}\right\}^{2} \frac{\left(\theta_{1}\left(\theta_{1}-1\right)\right)^{j}\left(\theta_{2}\left(\theta_{2}-1\right)\right)^{k-j}}{(j !)^{2}((k-j) !)^{2}} \\
= & \sum_{k=1}^{\infty} \sum_{j=0}^{k}\left\{\left(\begin{array}{l}
k \\
j
\end{array}\right)\right\}^{2}\left\{\frac{p_{2} q_{1}}{\left(p_{1}+p_{2}-p_{1} p_{2}\right)^{(k+1)}}-\frac{j}{k\left(p_{1}+p_{2}-p_{1} p_{2}\right)^{k}}\right\}^{2} \\
& \times\left(p_{1}^{2} q_{2}\right)^{k-j}\left(p_{2}^{2} q_{1}\right)^{j}
\end{aligned}
$$

Murthy (1956) derived the $k$ th Bhattacharyya lower bounds for the variance of an unbiased estimator for the geometric r.v. He differentiated $\tau(\theta)$ with respect to $p=\theta^{-1}$ instead of with respect to $p^{-1}=\theta$. In that case, the determination of the Bhattacharyya bounds will be more complicated since the Bhattacharyya matrix is not diagonal. Table 3 contains the values of some generalized Bhattacharyya bounds for different values of $p_{1}$ and $p_{2}$. It is clear that the convergence is fairly fast and as $k$ gets larger, there is a further improvement. We can also see some bounds are equal to the exact variance $\sigma^{2}(\widehat{\tau})$ (to the degree of approximation that we have used).

TABLE 3

Generalized Bhattacharyya bounds for the best unbiased estimator of $P(Y<X)$ in the geometric case

\begin{tabular}{rcccccccr}
\hline$p_{1}$ & $p_{2}$ & $\sigma^{2}(\widehat{\tau})$ & $B_{1}$ & $B_{2}$ & $B_{3}$ & $B_{10}$ & $B_{15}$ & $B_{20}$ \\
\hline .40 & .50 & .2448 & .1299 & .1665 & .1851 & .2266 & .2338 & .2338 \\
.60 & .70 & .2169 & .1318 & .1682 & .1858 & .2142 & .2161 & .2162 \\
.80 & .90 & .1499 & .1147 & .1338 & .1456 & .1499 & .1499 & .1499 \\
.70 & .50 & .1453 & .0810 & .1066 & .1191 & .1417 & .1442 & .1447 \\
.90 & .70 & .0670 & .0462 & .0587 & .0633 & .0668 & .0670 & .0670 \\
\hline
\end{tabular}


Acknowledgements. I wish to express my sincere thanks to Dr. D. N. Shanbhag for his helpful comments and suggestions in the preparation of this note.

\section{References}

A. A. Alzaid (1983), Some contributions to characterization theory, Ph.D. Thesis, Sheffield University.

A. A. Alzaid (1987), A note on the Meixner class, Pakistan J. Statist. 3, 79-82.

J. Bartoszewicz (1980), On the convergence of Bhattacharyya bounds in the multiparameter case. Zastos. Mat. 16, 601-608.

A. A. Bhattacharyya (1947), On some analogues of the amount of information and their use in statistical estimation, Sankhyā Ser. A 8, 201-218.

Z. W. Birnbaum (1956), On a use of the Mann-Whitney statistic, in: Proc. Third Berkeley Sympos. Math. Statist. Probab. 1, 13-17.

B. J. N. Blight and P. V. Rao (1974), The convergence of Bhattacharyya bounds, Biometrika 61, 137-142.

J. D. Church and B. Harris (1970), The estimation of reliability from stress-strength relationships, Technometrics 12, 49-54.

F. Downton (1973), The estimation of $\operatorname{Pr}(Y<X)$ in the normal case, ibid. 15, 551-558.

J. K. Ghosh and Y. S. Sathe (1987), Convergence of the Bhattacharyya boundsrevisited, Sankhyā Ser. A 49, 37-42.

Z. Govindarajulu (1968), Distribution-free confidence bounds for $P(X<Y)$, Ann. Inst. Statist. Math. 20, 229-238.

N. L. Johnson (1975), Letter to the editor, Technometrics 17, 393

G. D. Kelley et al. (1976), Efficient estimation of $P(Y<X)$ in the exponential case, ibid. 18, 359-360.

R. A. Khan (1984), On UMVU estimators and Bhattacharyya bounds in exponential distributions, J. Statist. Plann. Inference 9, 199-206.

R. A. Murthy (1956), A note on Bhattacharyya bounds for negative binomial distribution, Ann. Math. Statist. 27, 1182-1183.

D. B. Owen et al. (1964), Nonparametric upper confidence bounds for $\operatorname{Pr}(Y<X)$ and confidence limits for $\operatorname{Pr}\{Y<X\}$ when $X$ and $Y$ are normal, J. Amer. Statist. Assoc. 59, 906-924.

B. Reiser and I. Guttman (1986), Statistical inference for $\operatorname{Pr}(Y<X)$ : The normal case, Technometrics 28, 253-257.

B. Reiser and I. Guttman (1987), A comparison of three point estimators for $P(Y<X)$ in the normal case, Comput. Statist. Data Anal. 5, 59-66.

G. R. Seth (1949), On the variance of estimates, Ann. Math. Statist. 20, 1-27.

D. N. Shanbhag (1972), Some characterizations based on the Bhattacharyya matrix, J. Appl. Probab. 9, 580-587.

D. N. Shanbhag (1979), Diagonality of the Bhattacharyya matrix as a characterization, Theory Probab. Appl. 24, 430-433.

H. Tong (1974), A note on the estimation of $\operatorname{Pr}\{Y<X\}$ in the exponential case, Technometrics 16,625 . 
H. Tong (1975), Errata: A note on the estimation of $\operatorname{Pr}\{Y<X\}$ in the exponential case, ibid. 17, 395.

ABDULGHANi A. G. ALHARBI

DEPARTMENT OF PHYSICS \& MATHEMATICS

UNIVERSITY OF KING ABDULAZIZ

P.O. BOX 344

MADINAH MUNAWWARAH

SAUDI ARABIA

Received on 14.6.1993;

revised version on 26.11 .1993 